\title{
Investigating the Discrimination Ability of 3D Convolutional Neural Networks Applied to Altered Brain MRI Parametric Maps
}

This paper was downloaded from TechRxiv (https://www.techrxiv.org).

\section{LICENSE}

CC BY-NC-SA 4.0

SUBMISSION DATE / POSTED DATE

$19-07-2021 / 19-07-2021$

\section{CITATION}

Mattia, Giulia Maria; Nemmi, Federico; Villain, Edouard; Le Lann, Marie-Véronique; Franceries, Xavier; Péran, Patrice (2021): Investigating the Discrimination Ability of 3D Convolutional Neural Networks Applied to Altered Brain MRI Parametric Maps. TechRxiv. Preprint. https://doi.org/10.36227/techrxiv.15010803.v1

$\mathrm{DOI}$ 


\title{
Investigating the Discrimination Ability of 3D Convolutional Neural Networks Applied to Altered Brain MRI Parametric Maps
}

\author{
Giulia Maria Mattia, Federico Nemmi, Edouard Villain, Marie-Véronique Le Lann, Xavier Franceries and \\ Patrice Péran
}

\begin{abstract}
Convolutional neural networks are gradually being recognized in the neuroimaging community as a powerful tool for image analysis. In the present study, we tested the ability of 3D convolutional neural networks to discriminate between whole-brain parametric maps obtained from diffusion-weighted magnetic resonance imaging. Original parametric maps were subjected to intensity-based regionspecific alterations, to create altered maps. To analyze how position, size and intensity of altered regions affected the networks' learning process, we generated monoregion and biregion maps by systematically modifying the size and intensity of one or two brain regions in each image. We assessed network performance over a range of intensity increases and combinations of maps, carrying out 10-fold cross-validation and using a hold-out set for testing. We then tested the networks trained with monoregion images on the corresponding biregion images and vice versa. Results showed an inversely proportional link between size and intensity for the monoregion networks, in that the larger the region, the smaller the increase in intensity needed to achieve good performances. Accuracy was better for biregion networks than for their monoregion counterparts, showing that altering more than one region in the brain can improve discrimination. Monoregion networks correctly detected their target region in biregion maps, whereas biregion networks could only detect one of the two target regions at most. Biregion networks therefore learned a more complex pattern that was absent from the monoregion images. This deep learning approach could be tailored to explore the behavior of other convolutional neural networks for other regions of interest.
\end{abstract}

Index Terms-Artificial Intelligence, Brain Biomarkers, Convolutional Neural Network, Deep Learning, Medical Image Classification, Simulated Images

\section{INTRODUCTION}

Giulia Maria Mattia, Federico Nemmi and Patrice Péran: ToNIC, Toulouse Neurolmaging Center, Université de Toulouse, Inserm, UPS, France (e-mail: giulia-maria.mattia@inserm.fr, federico.nemmi@inserm.fr, patrice.peran@inserm.fr)

Edouard Villain: System Analysis and Architecture Laboratory (LAAS), Toulouse Paul Sabatier University-CNRS-INSA-INSERM, Toulouse, France and ToNIC, Toulouse Neurolmaging Center, Université de Toulouse, Inserm, UPS, France (e-mail: evillain@laas.fr)

Xavier Franceries: Toulouse Cancer Research Center (CRCT), UMR 1037, INSERM, Toulouse, France (e-mail: xavier.franceries@inserm.fr)

Marie Véronique Le Lann: LAAS, Toulouse Paul Sabatier UniversityCNRS-INSA, Toulouse, France (e-mail: mvlelann@laas.fr)
C ONVOLUTIONAL neural networks (CNNs) are increasingly being used in medical image analysis for a range of tasks, including classification and segmentation [1]. CNNs have shown outstanding performances for classifying neurological and neurodegenerative disorders [2], [3] such as Alzheimer's disease (AD) [4] and Parkinson's disease (PD) [5]). These networks can directly process original data, thereby freeing researchers from time-consuming manual feature extraction. Made up of multiple nonlinear modules that can create representations at simple but also very abstract levels, CNNs allow features to be automatically extracted during the learning phase [6]. Current advances in computational resources mean that $3 \mathrm{D}$ images could henceforth be used as input for CNNs. These have several advantages over 2D images [7]-[9]. In particular, 3D CNN architectures are able to integrate spatial information from the whole brain, unlike 2D brain slices [10].

Despite their remarkable performances, CNNs are regarded as black boxes, owing to their nontransparent decision-making process and their low interpretability, which hinders their usage [11], [12]. To improve this interpretability, various techniques have been designed to produce explanations at the processing level (e.g. GradCAM [13], saliency maps [14]), or representations of different network components (i.e. layers, units) along with the creation of self-explainable models [15]. However, all these explanations remain marginal, as they always refer to CNN subparts [12]. As an alternative, we suggest describing CNNs in terms not only of their architecture, learning rules and objective functions, but also the data they analyze [16]. The more complex the input data, the more difficult it may be to interpret the outcome, as CNNs learn from these data. Thus, if the input has known characteristics, then there may be a predictable output. Biomedical data are generally heterogeneous, mainly owing to interindividual variability. This complicates the study of rare diseases, for which only small samples are available. Understanding how the outcome changes according to the input is pivotal, if we wish specific patterns to emerge from the learning procedure. This is particularly challenging if the input data are 3D images containing multiple and diverse structures, as in the case of magnetic resonance imaging (MRI) of the brain, which can provide both structural and functional information about intracranial conditions [17]. 
In the present study, we used modified brain MRI data to study the behavior of 3D CNNs, and more specifically how their discrimination ability varies according to input. First, we altered parametric maps derived from diffusion-weighted MRI, by modifying the intensity of specific regions. We refer to these images hereafter as altered parametric maps (APMaps), and to the original parametric maps as OPMaps. Diffusion-weighted imaging (DWI) is commonly used to extract parameters measuring the Brownian motion of water molecules [18] which can reflect microstructural integrity, as in the case of mean diffusivity (MD) maps [19], [20]. MD increases related to pathophysiological changes have been already observed in Alzheimer's disease [21], Parkinson's disease [22] and multiple system atrophy (MSA) [23].

To create the APMaps from the OPMaps, we focused on two specific regions: the cerebellum and putamen. These brain regions have highly dissimilar characteristics, in term of size, tissue composition, shape, and location within the brain. In addition, the cerebellum and putamen are key regions for the assessment of a plethora of brain diseases, including motor disorders and cognitive dysfunctions [24]-[26]. For instance, cerebellar ataxia and putaminal alterations are both encountered in multiple system atrophy, categorized as an atypical Parkinsonian syndrome (ATP) [23], [27]-[30]. Indeed, biomarkers extracted from the putamen are currently under development to distinguish Parkinson's disease from atypical syndromes [31].

Using these two anatomical regions, we examined how size, intensity (i.e. value of parametric map), and position affected CNN performance. These features are crucial, as they define the patterns of brain modifications related to the pathological changes that occur as a disease progresses or across pathologies. More specifically, regarding intensity modification, our APMaps could be thought of as images simulating unhealthy patterns that become more evident as the intensity increases, as high MD values can indicate a pathological condition. We can therefore assume that small increases in intensity correspond to early pathological states that are more similar to healthy states and thus more difficult to tell apart, whereas large increases in intensity tend to mimic advanced states that are easier to spot. While minimizing the complexity of the input, we attempted to simulate real-life situations that might be encountered in clinical practice, creating more easily interpretable yet realistic 3D brain data. Our aim was to study the discrimination ability of 3D CNNs when dealing with region-specific APMaps, in order to better understand how salient features of the input can affect performance. We devised an innovative deep learning approach to investigate how the information contained in neuroimaging data can influence a network's ability to learn specific patterns corresponding to healthy or pathological states.

\section{Materials And Methods}

\section{A. Dataset}

1) Participants and MRI Protocol: Participants (89 men) underwent brain imaging in a 3T MRI scanner (Philips

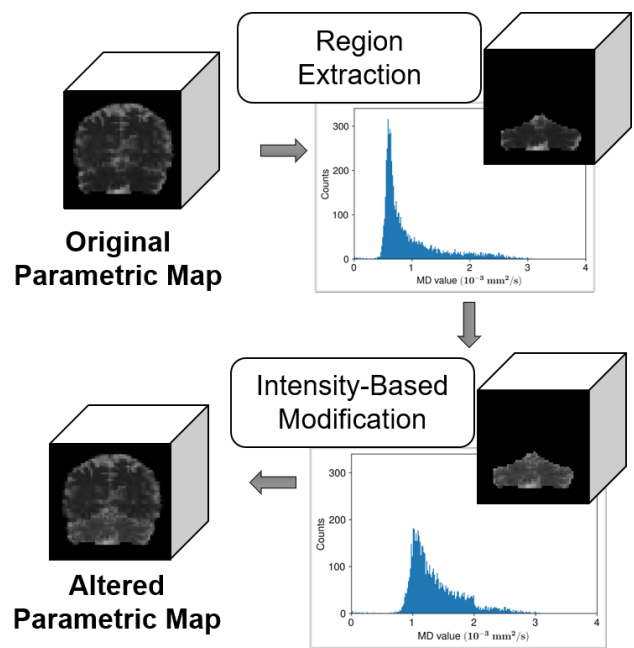

Fig. 1. Schematic representation of the proposed method for creating altered brain MRI parametric maps

Achieva) with a 32-channel head coil at the INSERM/UPS UMR1214 ToNIC technical platform (Toulouse, France). DWI acquisition parameters were as follows: $\mathrm{TE}=55 \mathrm{~ms}$; $\mathrm{TR}=$ $12.36 \mathrm{~s} ;$ flip angle $=90^{\circ} ; \mathrm{FOV}=112 \times 112$ voxels; number of slices $=65$; voxel size $=2 \times 2 \times 2 \mathrm{~mm}^{3}$; EPI factor $=59$; parallel factor $=2$; phase encoding direction $=$ postero-anterior; $b$ value (number of directions) $=$ $0(1), 500(32), 1000(32) \mathrm{s} / \mathrm{mm}^{2}$; total acquisition time $=$ 16 min $0 \mathrm{~s}$.

Participants' mean age was 56.19 years $(\mathrm{SD}=18.08$, range $=20.67-85.25)$.

2) Image Preprocessing: DW images were processed with the standard FSL pipeline [32]. We computed mean diffusivity maps and registered them in Montreal NeurologicaI Institute space with a $3 \times 3 \times 3 \quad \mathrm{~mm}^{3}$ resolution, using nonlinear registration.

3) Creation of Altered Parametric Maps: We developed a method of modifying MRI parametric maps of healthy brains by introducing region-specific alterations. To this end, we applied a linear intensity-based transformation to specific brain regions of the MD maps: increased MD values generally indicate water diffusion anomalies, suggesting reduced microstructural integrity [18].

We chose the cerebellum and putamen as regions of interest, as they differ in several respects:

- position in the brain: the cerebellum is located underneath the brain hemispheres, surrounded by gray matter dorsally (the occipital lobe), and the meninges and cerebrospinal fluid ventrally and posteriorly. By contrast, the putamen is located at the base of the forebrain, surrounded mainly by white matter;

- morphology: the cerebellum is a single rounded structure, whereas the putamen, though rounded, is bilateral;

- size: in normal individuals, the cerebellum has a mean volume of $300 \mathrm{~cm}^{3}$ [33], whereas the putamen has a mean volume of around $3.60 \mathrm{~cm}^{3}$ [34];

- tissue composition: there is considerable heterogeneity in the cerebellum, owing to the presence of gray and white 


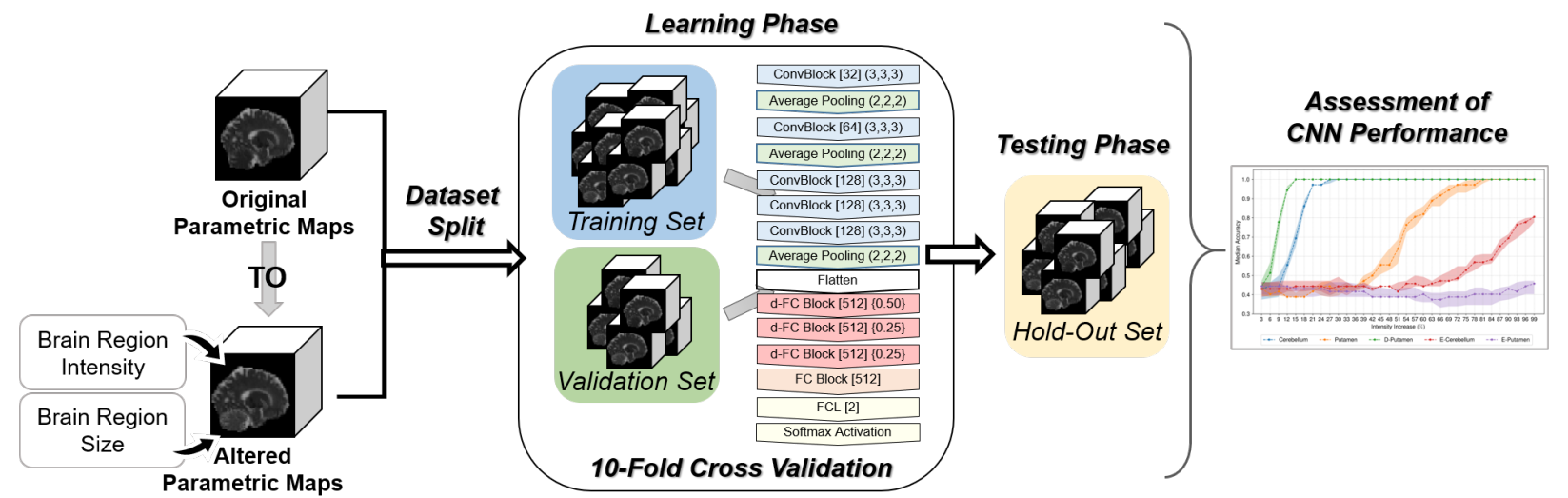

Fig. 2. Main steps of our investigatory approach: MRI parametric maps of healthy individuals are modified to create the altered parametric maps, via specific intensity modifications and size changes according to particular region(s) of interest. The original and altered parametric maps are split into three nonoverlapping sets: a training set and validation set derived from a 10 -fold cross-validation scheme, fed to a convolutional neural network to perform a binary classification task (original vs altered parametric maps), and a hold-out set for the testing phase. All the alterations made to the original parametric images can help assess how CNN performance varies according to input

matter along with cerebrospinal fluid. The putamen is essentially composed of gray-matter tissue, thus making it quite homogeneous.

The main steps for creating APMaps are summarized in Fig. 1. Regions of interest were extracted from the brain volumes of normal individuals using an atlas [35]. Only voxels within these regions underwent the intensity modification, leaving the rest of each image unaltered.

The modifications to regional intensity can be modeled as

$$
y_{r, n}=(1+p) x_{r, n}
$$

where $y_{r, n}$ and $x_{r, n}$ represent altered and original regions $(r)$, whose MD values lie below the $n^{t h}$ percentile $(n)$, and $p$ indicates the intensity increase as a percentage. Percentages ranged from $3 \%$ to $99 \%$, in increments of $3 \%$. The $n^{\text {th }}$ percentile was selected from three percentiles (i.e. $75^{t h}, 90^{t h}$, and $100^{t h}$ ) to limit image saturation effects, leading to the $75^{\text {th }}$ percentile being chosen for the cerebellum and the $90^{\text {th }}$ for the putamen, supported by visual assessment of the newly created images. Additional details and examples of APMaps are available in Supplementary Materials ${ }^{1}$.

To assess the impact of region size relative to position on CNN performance, we performed morphological operations on the anatomical brain region masks: we eroded the cerebellum (E-Cerebellum) to match the putamen's size and dilated the putamen (D-Putamen) to match that of the cerebellum. In this way, we modified the size of each region while preserving its anatomical position. Reference values for region size in terms of voxel number in the considered images are averaged around 6300 for the cerebellum and 400 for the putamen.

We produced monoregion APMaps, where one region was subjected to intensity modification, and biregion APMaps, where two regions underwent intensity modification. The latter are thoroughly described in the following sections.

\footnotetext{
${ }^{1}$ For Supplementary Materials, see supporting documents/multimedia tab.
}

\section{B. Convolutional Neural Networks}

Convolutional neural networks allow for automatic feature extraction of multiple arrays (e.g. 3D images), usually used for classification tasks via multilayer artificial neural networks [6]. Just like the processing core of a neural network, an artificial neuron is able to compute an output (e.g. predicted class of the input in supervised classification), using the parameters learned during training to minimize a loss function. The mathematical operations needed to compute the output $c$ are described in (2): an activation function $(f)$ is applied to the sum of a bias term $g$ and the weights $w$, multiplied by each input $i$.

$$
c=f\left(\sum_{j} i_{j} w_{j}+g\right)
$$

A major advance in artificial neural networks has been the introduction of the local receptive field of the artificial neuron, inspired by Hubel and Wiesel's study on the cat's visual cortex [36], [37]. This replaces the traditional fully connected scheme of a neural network, which would require too many parameters were images used as input. A single neuron is therefore in charge of scanning the input, and its resulting states are stored in the corresponding positions of a layer, referred to as a feature map. The latter corresponds to a convolution operation using a kernel of small dimensions, whose output undergoes a sub-sampling operation performed by pooling. This decreases the resolution of the feature map and results in lessened sensitivity to distortions and shifts [38]. In a supervised classification task, learning is accomplished by the back-propagation of errors between predictions and ground truth throughout the network [39].

In the present study, we devised a 3D CNN for supervised binary classification, the task being to distinguish original brain MRI parametric maps from altered ones. Feeding the entire brain volume into a $\mathrm{CNN}$ as input preserves the spatial information of the whole MRI at a 3D participant level [40]. An overall representation of the deep learning approach implemented in this study is illustrated in Fig. 2. The CNNs received input in the shape of 89 OPmaps and 89 APMaps. 
Given the limited sample size, we carried out cross-validation during the learning phase, as is customary in the neuroimaging field [40], [41]. Each dataset (i.e. 178 images) was randomly divided: $80 \%$ to be used in 10 -fold cross-validation during the learning phase, and the remaining $20 \%$ to be used as a holdout set to assess CNN performance in the testing phase. The random seed for cross-validation was kept constant. The best epoch model with minimum loss value on the validation set was selected to establish network parameters and be tested on the hold-out set.

The CNN architecture described here was inspired by AlexNet [42] and VGG-Net [43]. A schematic diagram representing our model is provided in Fig. 3 showing the following building blocks:

- ConvBlock, composed by a convolutional layer characterized by a $3 \times 3 \times 3$ filter size, stride $=1$, with an increasing number of kernels going deeper in the network, and a batch normalization (BN) layer to speed up learning through a reduction in internal covariate shift [44], and an exponential linear unit (ELU) as the activation function [45];

- Average Pooling, to retain as much information as possible throughout the network, with a filter size of $2 \times 2 \times 2$ and stride $=2$;

- $d-F C$ Block, including a fully connected layer with 512 neurons to ensure enough units are available for final classification, followed by BN layer, ELU activation and a dropout layer in a regularization technique intended to prevent overfitting [46];

- FC Block, same as d-FC Block but without dropout;

- $F C L$, fully connected layer for binary classification with two neurons followed by softmax activation function.

The CNNs were implemented using Keras library version 2.2.4 [47] and TensorFlow library version 1.13.1 [48] with the support of an Nvidia ${ }^{\circledR}$ Quadro RTX 6000 graphical processing unit.

L2 regularization was applied with a factor of 0.0005 along with the valid method in convolutional layers to avoid padding [47]. The model trained over 100 epochs with an initial learning rate of 0.00001 , subject to dynamic reduction if there was no improvement in performance after five epochs. Training was carried out using mini-batch gradient descent, with a batch size of eight samples to meet computational requirements. Categorical cross-entropy (i.e. logarithmic loss function) was used together with Adam optimizer, which is characterized by the combination of an adaptive gradient algorithm with root mean square propagation and is especially suited to problems involving large amounts of data [49].

To compare performances across models, the chosen metric was defined as

$$
\text { Accuracy }=\frac{T P+T N}{T P+F N+T N+F P},
$$

where $T P$ stands for true positive (i.e. samples correctly identified as belonging to the positive class (APMaps in our case study)), $T N$ for true negative (i.e. OPMaps correctly classified as such), $F N$ for false negative (i.e. APMaps mistaken for OPMaps), and $F P$ for false positive (i.e. OPMaps mistaken for

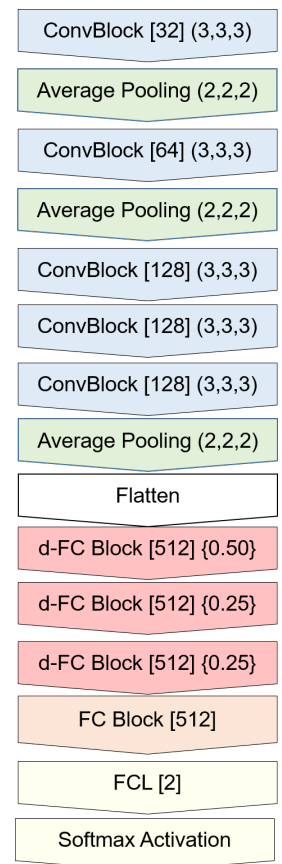

(a) Architecture

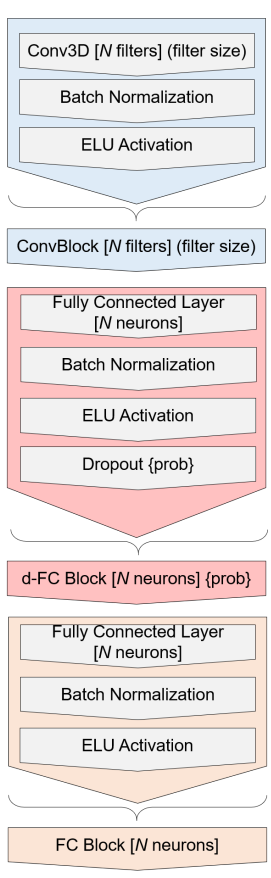

(b) Building blocks
Fig. 3. Schematic diagram of the convolutional neural network architecture and its building blocks. BN: batch normalization; ELU: exponential linear unit; FC: fully connected; FCL: fully connected layer; prob: dropout probability

APMaps). Accuracy for each model was provided as median and interquartile range (IQR) achieved on the hold-out set, given the adoption of 10 -fold cross validation.

\section{Assessment of CNN Performance}

CNN performance was first assessed using OPMaps and monoregion APMaps (intensity from 3\% to 99\%) as input. The results of this assessment allowed us to identify the set of intensity increases that were clinically meaningful, based on each model's level of accuracy. Biregion APMaps were obtained by applying the above-mentioned method to each region concerned. We combined regions on the basis of their size and the accuracy levels of the CNNs trained with monoregion APMaps. For brevity's sake, we define monoregion and biregion CNNs according to the input data used in the performance assessment (i.e. OPMaps with monoregion or biregion APMaps).

Biregion parametric maps were characterized by two modified regions, which were paired according to size: either different (i.e. cerebellum/putamen) or comparable (i.e. DPutamen/cerebellum or E-Cerebellum/putamen).

We established four levels of accuracy representative of clinically significant situations: very low (VL), low (L), fair (F), and high $(\mathrm{H})$, whose reference values are $0.45,0.65,0.85$, and 1.00. Monoregion CNNs were assigned the level closest to the accuracy value they achieved. If needed, additional intensity increases in $1 \%$ increments were computed to comply as precisely as possible with the four levels of accuracy. Interested readers can find these results in the Supplementary Materials. When the same accuracy value (e.g. equal to 1.00) 


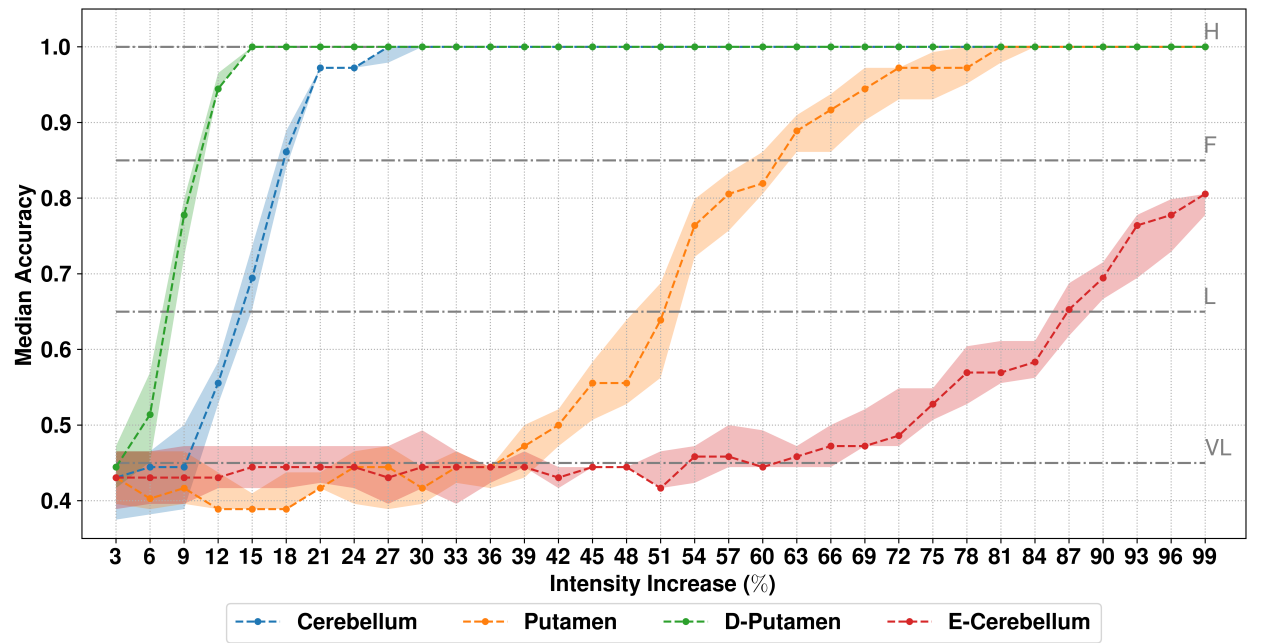

Fig. 4. Monoregion CNNs. Accuracy on hold-out set given as median (IQR) over 10 folds with intensity increases in altered parametric maps. Gray lines indicate the four accuracy levels used for performance assessment. D: dilated; E: eroded; F: fair; H: high; L: low; VL: very low

corresponded to multiple intensity increases, we selected the one with the highest minimum accuracy across all 10 folds. Each accuracy level corresponded to a particular intensity increase that was applied to the OPMaps of every region to be modified to create biregion APMaps.

We looked at all 16 possible combinations of accuracy levels, either the same (i.e. VL/VL, L/L, F/F, H/H) or different (e.g. $\mathrm{VL} / \mathrm{L}, \mathrm{L} / \mathrm{F}$ ) between regions, to evaluate the relative effect of position and size.

Moreover, we compared monoregion with biregion CNNs by testing monoregion CNNs on biregion APMaps and vice versa to understand the contribution that each accuracy level made to the learned patterns. To this end, we implemented:

- biregion CNNs trained with biregion images of $\mathrm{H} / \mathrm{H}$ accuracy combination and tested on monoregion APMaps with intensity increases dictated by the corresponding $\mathrm{H}$ level of accuracy;

- monoregion CNNs trained with monoregion images with intensity increases dictated by the $\mathrm{H}$ level of accuracy and tested on the corresponding biregion APMaps with the $\mathrm{H} / \mathrm{H}$ accuracy combination.

\section{Results}

\section{A. Monoregion CNNs}

Our investigation of $\mathrm{CNN}$ discrimination ability began with the assessment of discrimination performances between OPMaps and APMaps, the latter modified over a range of intensity increases. Fig. 4 shows the changes in CNN performance, reporting the median accuracy (and IQR) achieved on the hold-out set for each increase in intensity percentage applied to the regions. Cerebellum and D-Putamen models exhibited similar behavior, even though the former reached maximum accuracy at $27 \%$, compared with $15 \%$ for the latter. The putamen model yielded accuracy of 1.00 at $84 \%$, whereas the E-Cerebellum model only reached accuracy of 0.81 , for an intensity increase of $99 \%$. Despite the equivalent region size, the improvement in accuracy across intensity was much slower for the E-Cerebellum than for the putamen, and the chancelevel thresholds (i.e. to achieve accuracy equal to 0.50) were very high ( $87 \%$ and $51 \%)$.

\section{B. Biregion CNNs}

Biregion CNNs were trained to distinguish OPMaps from biregion APMaps, in which two regions had undergone intensity-based modification. Bar plots depicted in Fig. 5 represent the accuracy achieved by the biregion CNNs compared with the best accuracy reached by the monoregion CNNs (between the two considered accuracy levels). $T$ tests computed between biregion accuracy and best monoregion accuracy showed that biregion performance was significantly better for the VL/VL, L/L and F/F combinations.

All three pairs of regions showed outstanding performance (accuracy equal to 1.00) when at least of the two regions was characterized by the $\mathrm{H}$ accuracy level.

For mixed combinations with F, L and VL, the biregion CNNs significantly outperformed their monoregion counterparts. However, there were no significant differences for the F/VL (D-Putamen/cerebellum) and VL/F (cerebellum/putamen) combinations.

Using a one-way analysis of variance (ANOVA), we identified meaningful combinations for each biregion performance between the levels of accuracy represented by blue, orange, or green bars in Fig. 5. Accuracy was significantly lower for VL/VL than for other combinations, and all the other comparisons (e.g. VL/L vs. L/VL, VL/F vs. F/VL) revealed smaller differences in accuracy.

For the sake of clarity, significant differences derived from the one-way ANOVA are not shown in Fig. 5, but are listed in Supplementary Materials.

\section{Monoregion vs Biregion CNNs}

Monoregion CNNs were used to discriminate OPMaps from biregion APMaps, and biregion CNNs had to distinguish OPMaps from monoregion APMaps, matching intensity 


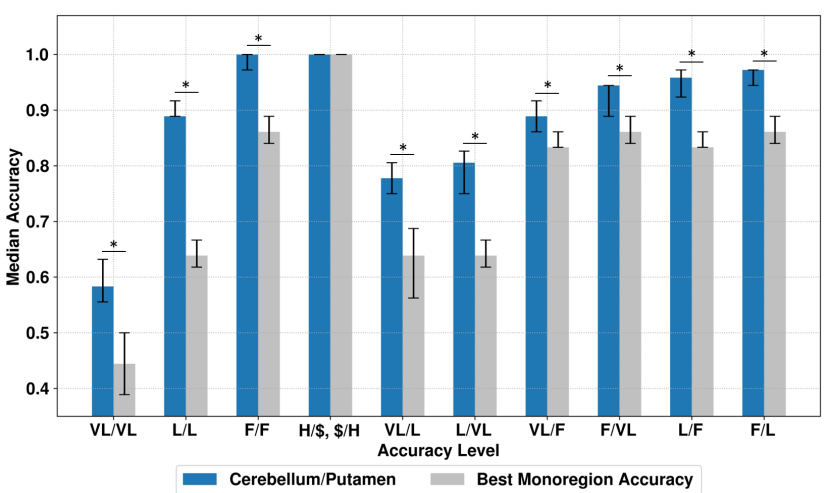

(a) Cerebellum/Putamen

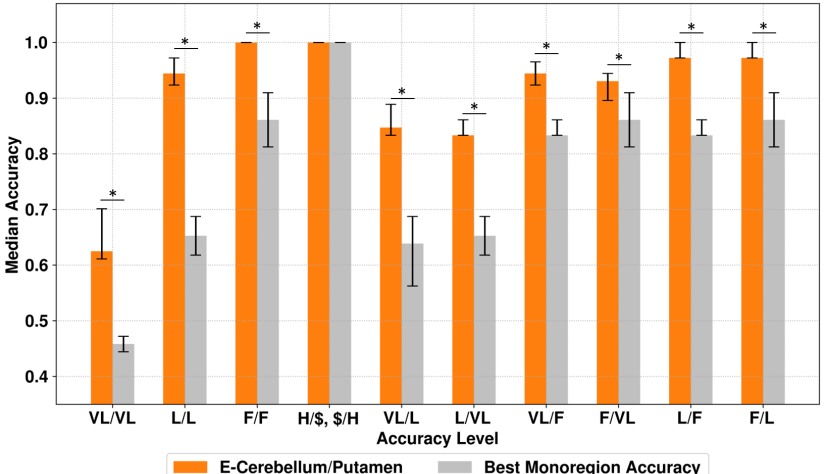

(b) E-Cerebellum/putamen

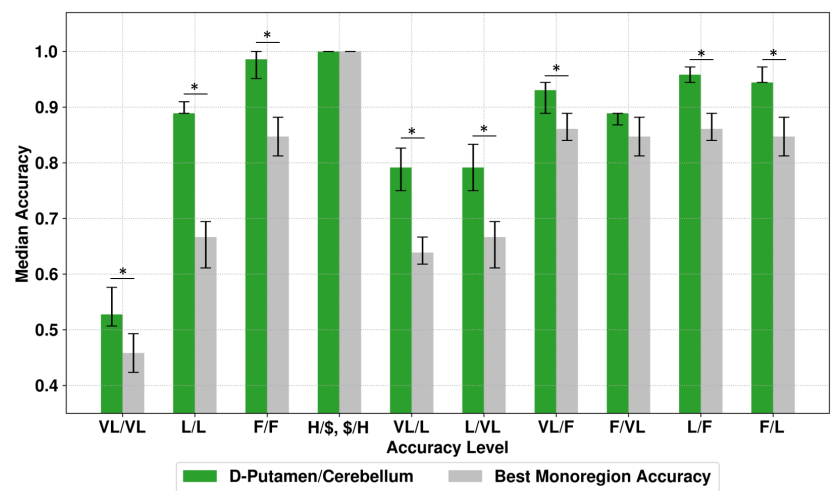

(c) D-Putamen/Cerebellum

Fig. 5. Biregion CNNs. Median (IQR) accuracy on hold-out set over 10 folds compared with the best monoregion performance for the $\mathbf{1 6}$ combinations of accuracy levels. The dollar sign stands for $\mathrm{VL}, \mathrm{L}, \mathrm{F}, \mathrm{H}$, as all combinations featuring $\mathrm{H}$ had equal performances. $* \boldsymbol{p}<\mathbf{0 . 0 5}$; D: dilated; E: eroded; F: fair; H: high; L: low; VL: very low

increase between regions in the monoregion and biregion APMaps. We can see in Table I that monoregion CNNs successfully recognized the relevant altered regions in each biregion image, achieving maximum median accuracy for each one of them, except for E-Cerebellum and D-Putamen, where median accuracy was slightly lower (0.97). By contrast,

TABLE I

Monoregion vs Biregion CNNs: CNNS TRAINED WITH MONOREGION APMAPS CORRESPONDING TO H ACCURACY LEVEL WERE TESTED ON THE CORRESPONDING H/H BIREGION HOLD-OUT SET AND VICE-VERSA. ACCURACY IS PROVIDED AS MEDIAN (IQR) OVER 10 FOLDS. D: DILATED; E: ERODED; F: FAIR; H: HIGH; L: LOW; VL: VERY LOW

\begin{tabular}{|c|c|c|}
\hline Training & Testing & \\
\hline Monoregion CNN & Biregion APMaps & Accuracy \\
\hline Cerebellum & \multirow{2}{*}{ Cerebellum/Putamen } & $1.00(0.00)$ \\
\hline Putamen & & $1.00(0.00)$ \\
\hline E-Cerebellum & \multirow{2}{*}{ E-Cerebellum/putamen } & $0.97(0.03)$ \\
\hline Putamen & & $1.00(0.00)$ \\
\hline D-Putamen & \multirow{2}{*}{ D-Putamen/Cerebellum } & $0.97(0.03)$ \\
\hline Cerebellum & & $1.00(0.00)$ \\
\hline Biregion CNN & Monoregion APMaps & Accuracy \\
\hline \multirow{2}{*}{ Cerebellum/Putamen } & Cerebellum & $0.97(0.02)$ \\
\hline & Putamen & $0.50(0.02)$ \\
\hline \multirow{2}{*}{ E-Cerebellum/putamen } & E-Cerebellum & $0.64(0.08)$ \\
\hline & Putamen & $0.65(0.03)$ \\
\hline \multirow{2}{*}{ D-Putamen/Cerebellum } & D-Putamen & $0.56(0.03)$ \\
\hline & Cerebellum & $0.89(0.10)$ \\
\hline
\end{tabular}

biregion CNNs performed poorly on some of their respective monoregion test images. The biregion $\mathrm{CNN}$ trained with the D-Putamen/cerebellum APMaps successfully classified the cerebellum with a median accuracy of 0.89 , but performed poorly on D-Putamen. However, the biregion CNN trained with E-Cerebellum/putamen APMaps did not identify either the Putamen or the E-Cerebellum, only achieving a median accuracy of around 0.65 .

Regarding regions of different sizes, the cerebellum/putamen biregion CNN recognized almost to perfection the cerebellum with median accuracy of 0.97 , despite its near-chance level performance for the putamen.

\section{Discussion}

In this study, we explored determinants of 3D parametric maps that may influence the discrimination performances of a CNN. Unsurprisingly, the more the parametric maps were altered in terms of size and intensity, the better the networks' discrimination performance. However, anatomical position also appeared to have an influence on the final outcome, given the differences in performances between regions of comparable size (e.g. putamen and E-Cerebellum). Another interesting result was that the accuracy of CNNs trained with biregion images turned out to be systematically higher than the best accuracy for each region taken alone, even when monoregion accuracy was not satisfactory. Most remarkable was the case of the $\mathrm{L} / \mathrm{L}$ biregion combination, which exceeded 
monoregion accuracy of 0.65 by at least $23 \%$. Thus, although the single regions were not discriminant in isolation, together they provided a detectable pattern.

Looking at the performances of CNNs trained with monoregion APMaps with different intensity increases, we found a straightforward trend whereby the larger the region, the smaller the intensity increase required to achieve the highest accuracy. This inverse proportionality varied according to the position of the region inside the brain: being more centrally located, the putamen and D-Putamen outperformed their equally sized counterparts, E-Cerebellum and cerebellum, which were more on the periphery. CNNs seemed to need lower intensity increases to detect regions in the center of the brain rather than on its periphery, independently of the region's intrinsic heterogeneity (higher for cerebellum and D-Putamen). These results indicate that a larger and more central region with quite a low increase in parametric values compared with the original values (supposedly indicating an early pathological condition) can be distinguished more easily than smaller and more peripheral regions. Moreover, we determined an intensity threshold for each brain structure, to ensure a high level of accuracy for the proposed 3D CNN architecture. As we understood with the biregion approach, the information available when different accuracy levels were combined significantly improved performances, compared with when the regions were considered on their own.

In line with our expectations, when at least one region was featured with the $\mathrm{H}$ level of accuracy, biregion CNNs yielded equally excellent performances independently of region size. Unexpectedly, no difference was found between the mixed accuracy combinations, such as VL/L with respect to $\mathrm{L} / \mathrm{VL}$, suggesting that the knowledge available at each accuracy level was enough to boost performance regardless of region position and size.

One limitation of the present study was the restricted sample size, which came from the use of real-world neuroimaging data. Nevertheless, modifying the brain parametric maps of healthy individuals across a wide age range enabled us to preserve not only interindividual variability but also intrinsic heterogeneity in terms of morphology and anatomy, avoiding the distortions or artifacts that might arise from a completely artificial set.

Using monoregion APMaps to test biregion CNNs (and vice versa) revealed an intriguing behavior: performance deteriorated for at least one of the two regions and was poorer than that of the respective monoregion CNN. One possible explanation is that biregion CNNs learned a multispatial signature which could not be detected in the monoregion APMaps, hence the unsatisfactory performance on most of the regions singly. It is worth noting that the cerebellum was successfully classified by the biregion CNNs trained with biregion APMaps including the cerebellum, at the expense of the smaller (the putamen in cerebellum/putamen CNN) or more central region (the D-Putamen in D-Putamen/cerebellum CNN) region. This may indicate the predominance of one region over the other in the biregion retrieval pattern. By contrast, monoregion CNNs performed well on biregion APMaps. This is an interesting result from a clinical point of view. Neurodegenerative lesions start in a specific brain region and spread to other brain regions as the disease progresses [50]. A CNN trained with data derived from early pathological conditions (e.g. monoregion APMaps) should therefore be able to classify even advanced stages (e.g. biregion APMaps) involving the same region, whereas the opposite is less likely, as the learned pattern would be much more complex and feature a multispatial signature. Building region-specific CNNs may maximize the detection accuracy for abnormalities in a particular brain region.

All the results reported here are restricted to our specific 3D CNN and to two brain regions. However, our approach may pave the way to further investigations with different deep learning architectures and regions of interest, possibly even beyond the brain. These findings are just the starting point when it comes to grasping how the complexity of input data yielded by neuroimaging influences the pattern retrieval performed by CNNs. The general nature of the APMaps, in the sense that they were not intended to resemble any specific neuropathology, but just mimic an alteration that might conceivably be present in various brain diseases, may prove useful for recognizing region-specific abnormalities in real pathological images used as test sets.

Future developments could include the study of the minimum number of subjects needed to achieve reasonable performances, the use of visualization techniques to support $\mathrm{CNN}$ outcomes, and the creation of altered parametric maps resembling specific neuropathological states.

\section{ACKNOWLEDGMENT}

We would like to thank the MRI technical platform at Toulouse Neuroimaging Center (ToNIC), Université de Toulouse, Inserm, UPS, France.

The authors declare no conflict of interest.

\section{REFERENCES}

[1] G. Litjens et al., "A survey on deep learning in medical image analysis," Medical Image Analysis, vol. 42, pp. 60-88, 2017.

[2] S. Vieira, W. H. L. Pinaya, and A. Mechelli, "Using deep learning to investigate the neuroimaging correlates of psychiatric and neurological disorders: Methods and applications," Neuroscience \& Biobehavioral Reviews, vol. 74, pp. 58-75, 2017.

[3] M. B. T. Noor, N. Z. Zenia, M. Kaiser, M. Mahmud, and S. Mamun, "Detecting neurodegenerative disease from mri: A brief review on a deep learning perspective," in Brain Informatics, 2019.

[4] A. Payan and G. Montana, "Predicting Alzheimer's disease - a neuroimaging study with $3 \mathrm{~d}$ convolutional neural networks," ArXiv, vol. abs/1502.02506, 2015

[5] F. J. Martínez-Murcia et al., "A 3d convolutional neural network approach for the diagnosis of Parkinson's disease," in Natural and Artificial Computation for Biomedicine and Neuroscience (J. M. Ferrández Vicente, Á.-S. J. Ramón, F. de la Paz López, J. Toledo Moreo, and H. Adeli, eds.), (Cham), pp. 324-333, Springer International Publishing, 2017.

[6] Y. LeCun, Y. Bengio, and G. Hinton, "Deep learning," Nature, vol. 521, pp. 436-444, 2015.

[7] S. Esmaeilzadeh, Y. Yang, and E. Adeli, "End-to-end Parkinson disease diagnosis using brain mr-images by 3d-cnn," ArXiv, vol. abs/1806.05233, 2018.

[8] S. Sarraf, D. DeSouza, J. A. E. Anderson, and G. Tofighi, "DeepAD: Alzheimer's disease classification via deep convolutional neural networks using mri and fmri," bioRxiv, 2016.

[9] E. Hosseini-Asl, G. Gimel'farb, and A. El-Baz, "Alzheimer's disease diagnostics by a deeply supervised adaptable $3 \mathrm{~d}$ convolutional network," ArXiv, vol. abs/1607.00556, 2016. 
[10] E. Trivizakis et al., "Extending 2-d convolutional neural networks to 3-d for advancing deep learning cancer classification with application to mri liver tumor differentiation," IEEE Journal of Biomedical and Health Informatics, vol. 23, pp. 923-930, 2019.

[11] A. Shrikumar, P. Greenside, A. Shcherbina, and A. Kundaje, "Not just a black box: Learning important features through propagating activation differences," ArXiv, vol. abs/1605.01713, 2016.

[12] D. C. Elton, "Self-explaining ai as an alternative to interpretable ai," in Artificial General Intelligence (B. Goertzel, A. I. Panov, A. Potapov, and R. Yampolskiy, eds.), (Cham), pp. 95-106, Springer International Publishing, 2020.

[13] R. R. Selvaraju, A. Das, R. Vedantam, M. Cogswell, D. Parikh, and D. Batra, "Grad-cam: Visual explanations from deep networks via gradient-based localization," International Journal of Computer Vision, vol. 128, pp. 336-359, 2019.

[14] K. Simonyan, A. Vedaldi, and A. Zisserman, "Deep inside convolutional networks: Visualising image classification models and saliency maps," CoRR, vol. abs/1312.6034, 2014.

[15] L. H. Gilpin, D. Bau, B. Yuan, A. Bajwa, M. Specter, and L. Kagal, "Explaining explanations: An overview of interpretability of machine learning," 2018 IEEE 5th Int. Conf. on Data Science and Advanced Analytics (DSAA), pp. 80-89, 2018.

[16] B. A. Richards et al., "A deep learning framework for neuroscience," Nature Reviews Neuroscience, vol. 22, pp. 1761-1770, 2019.

[17] M. Rosenbloom and A. Pfefferbaum, "Magnetic resonance imaging of the living brain: Evidence for brain degeneration among alcoholics and recovery with abstinence.," Alcohol Research \& Health, vol. 31, pp. 362376, 2008.

[18] D. Bihan, "Looking into the functional architecture of the brain with diffusion mri," Nature Reviews Neuroscience, vol. 4, pp. 469-480, 2003.

[19] H. Kim, S. Kim, H. S. Kim, C. Choi, and C. Lee, "Alterations of mean diffusivity in brain white matter and deep gray matter in parkinson's disease," Neuroscience Letters, vol. 550, pp. 64-68, 2013.

[20] S. Vos, D. Jones, B. Jeurissen, M. Viergever, and A. Leemans, "The influence of complex white matter architecture on the mean diffusivity in diffusion tensor mri of the human brain," NeuroImage, vol. 59, pp. 22082216, 2012.

[21] P. Eustache, F. Nemmi, L. Saint-Aubert, J. Pariente, and P. Péran, "Multimodal magnetic resonance imaging in Alzheimer's disease patients at prodromal stage," Journal of Alzheimer's Disease, vol. 50, pp. 10351050, 2016.

[22] P. Péran et al., "Magnetic resonance imaging markers of Parkinson's disease nigrostriatal signature.," Brain, vol. 133, pp. 3423-3433, 08 2010.

[23] P. Péran et al., "Mri supervised and unsupervised classification of Parkinson's disease and multiple system atrophy," Movement Disorders, vol. 33, 2018.

[24] M. Molinari and M. Leggio, Cerebellum: Clinical Pathology, pp. 737742. Elsevier Ltd, 2010.

[25] N. Viñas-Guasch and Y. J. Wu, "The role of the putamen in language: a meta-analytic connectivity modeling study," Brain Structure and Function, vol. 222, pp. 3991-4004, 2017.

[26] S. Haber, "Corticostriatal circuitry," Dialogues in Clinical Neuroscience, vol. 18, pp. 7-21, 2016

[27] D. Berg, J. Steinberger, C. W. Olanow, T. Naidich, and T. Yousry, "Milestones in magnetic resonance imaging and transcranial sonography of movement disorders.," Movement Disorders, vol. 26 6, pp. 979-92, 2011.

[28] H. Shin, S. Kang, J. H. Yang, H. Kim, M.-S. Lee, and Y. Sohn, "Use of the putamen/caudate volume ratio for early differentiation between Parkinsonian variant of multiple system atrophy and Parkinson disease," Journal of Clinical Neurology (Seoul, Korea), vol. 3, pp. 79 - 81, 2007.

[29] K. Seppi et al., "Progression of putaminal degeneration in multiple system atrophy: A serial diffusion mr study," NeuroImage, vol. 31, pp. 240-245, 2006.

[30] G. Barbagallo et al., "Multimodal mri assessment of nigro-striatal pathway in multiple system atrophy and Parkinson disease," Movement Disorders, vol. 31 3, pp. 325-34, 2016.

[31] A. Michell, S. Lewis, T. Foltynie, and R. Barker, "Biomarkers and Parkinson's disease," Brain, vol. 127 Pt 8, pp. 1693-705, 2004.

[32] T. Behrens et al., "Characterization and propagation of uncertainty in diffusion-weighted $\mathrm{mr}$ imaging," Magnetic Resonance in Medicine, vol. 50 5, pp. 1077-88, 2003.

[33] G. Shepherd, The Synaptic Organization of the Brain, ch. 7. New York: Oxford University Press., 2004.
[34] D. Yin, F. Valles, M. Fiandaca, J. Forsayeth, and K. Bankiewicz, "Striatal volume differences between non-human and human primates," Journal of Neuroscience Methods, vol. 176, pp. 200-205, 2009.

[35] A. Hammers et al., "Three-dimensional maximum probability atlas of the human brain, with particular reference to the temporal lobe.," Human Brain Mapping, vol. 19 4, pp. 224-47, 2003.

[36] Y. LeCun et al., "Handwritten digit recognition with a back-propagation network," in NIPS, 1989.

[37] D. Hubel and T. Wiesel, "Receptive fields, binocular interaction and functional architecture in the cat's visual cortex," The Journal of Physiology, vol. 160, pp. 106-54, 1962.

[38] Y. Lecun, L. Bottou, Y. Bengio, and P. Haffner, "Gradient-based learning applied to document recognition," Proceedings of the IEEE, vol. 86, no. 11 , pp. 2278-2324, 1998.

[39] D. Rumelhart, G. E. Hinton, and R. J. Williams, "Learning representations by back-propagating errors," Nature, vol. 323, pp. 533-536, 1986.

[40] J. Wen et al., "Convolutional neural networks for classification of Alzheimer's disease: Overview and reproducible evaluation," Medical Image Analysis, vol. 63, p. 101694, 2020.

[41] M. I. Qureshi, J. Oh, and B. Lee, "3d-cnn based discrimination of schizophrenia using resting-state fmri," Artificial Intelligence in Medicine, vol. 98, pp. 10-17, 2019.

[42] A. Krizhevsky, I. Sutskever, and G. E. Hinton, "Imagenet classification with deep convolutional neural networks," in Communications of the ACM, 2017.

[43] K. Simonyan and A. Zisserman, "Very deep convolutional networks for large-scale image recognition," Computing Research Repository, vol. abs/1409.1556, 2015.

[44] S. Ioffe and C. Szegedy, "Batch normalization: Accelerating deep network training by reducing internal covariate shift," ArXiv, vol. abs/1502.03167, 2015.

[45] D.-A. Clevert, T. Unterthiner, and S. Hochreiter, "Fast and accurate deep network learning by exponential linear units (ELUs)," CoRR, vol. abs/1511.07289, 2016.

[46] N. Srivastava, G. E. Hinton, A. Krizhevsky, I. Sutskever, and R. Salakhutdinov, "Dropout: A simple way to prevent neural networks from overfitting," Journal of Machine Learning Research, vol. 15, pp. 1929-1958, 2014.

[47] F. Chollet et al., "Keras." https: / / keras.io, 2015.

[48] M. Abadi et al., "TensorFlow: A system for large-scale machine learning," in Proceedings of the 12th USENIX Symposium on Operating Systems Design and Implementation (OSDI), 2016.

[49] D. P. Kingma and J. Ba, "Adam: A method for stochastic optimization," CoRR, vol. abs/1412.6980, 2015.

[50] J. Brettschneider, K. Tredici, V. Lee, and J. Trojanowski, "Spreading of pathology in neurodegenerative diseases: A focus on human studies," Nature Reviews Neuroscience, vol. 16, pp. 109-120, 2015. 